\section{Análise de correspondência múltipla na avaliação de serviços de farmácia hospitalar no Brasil}

\author{
Multiple correspondence analysis in the evaluation \\ of hospital pharmacy services in Brazil
}

\author{
Análisis de correspondencia múltiple en la \\ evaluación de servicios de farmacia \\ hospitalaria en Brasil
}

Aline do Nascimento 1

Renan Moritz V. R. Almeida 1 Selma Rodrigues de Castilho 2 Antonio Fernando Catelli Infantosi 1

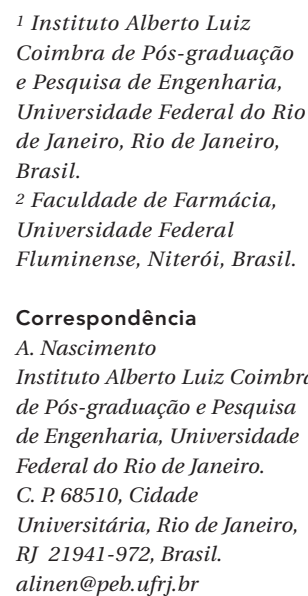

\begin{abstract}
This study aimed to evaluate associations among variables in hospital pharmacy services. Thirty variables were used from the project Diagnosis of Hospital Pharmacies in Brazil pertaining to the overall description of the hospital, overall characterization of the hospital pharmacy service, and stages in pharmaceutical care. The statistical techniques were multiple correspondence and cluster analysis. Dimension 1 of the multiple correspondence analysis explained $90.6 \%$ of variance, differentiating between hospital pharmacy services based on the presence of certain activities, thus suggesting an axis of characterization for the hospital pharmacy services. The results indicate a direct relationship between compliance with the activities and the type of hospital and pharmacists with specialized training. Cluster analysis identified six clusters related to hospital size; greater compliance with the activities was associated with large hospitals and those with a pharmacist (more time dedicated to the hospital pharmacy service and higher level of training). The study concludes that the techniques were able to identify associations and a concise range of variables for a comprehensive evaluation of hospital pharmacy services in Brazil.
\end{abstract}

Hospital Pharmacy Service; Pharmaceutical Services; Pharmaceutical Preparations

\section{Resumo}

O estudo objetivou avaliar a existência de associações entre variáveis de serviços de farmácia hospitalar. Foram utilizadas 30 variáveis do projeto Diagnóstico da Farmácia Hospitalar no Brasil relativas à caracterização geral do hospital, caracterização geral do serviço de farmácia hospitalar e etapas da assistência farmacêutica. A análise de correspondência múltipla e a análise de agrupamentos foram utilizadas. A dimensão 1 da análise de correspondência múltipla explicou 90,6\% da variabilidade, diferenciando os serviços de farmácia hospitalar conforme a presença de atividades, sugerindo assim um eixo de caracterização da estrutura dos serviços de farmácia hospitalar. Os resultados indicam relação direta entre cumprimento das atividades e tipo de hospital e farmacêuticos com especialização. A análise de agrupamentos identificou seis grupos relativos ao porte do hospital, tendo maior cumprimento de atividades os serviços de farmácia hospitalar em unidades de grande porte e com farmacêutico (maior tempo dedicado ao serviço de farmácia hospitalar e maior nível de treinamento). Conclui-se que as técnicas foram capazes de identificar as associações e um elenco conciso de variáveis para uma avaliação abrangente dos serviços de farmácia hospitalar no país.

Serviço de Farmácia Hospitalar; Assistência Farmacêutica; Preparações Farmacêuticas 


\section{Introdução}

O serviço de farmácia hospitalar é a unidade do hospital que desempenha atividades relacionadas aos medicamentos, integrado funcionalmente às demais unidades de assistência ao paciente 1. Esse serviço é extremamente importante no contexto assistencial, e tem como principal missão a provisão segura e racional de medicamentos, serviços e produtos para saúde. Tal objetivo é alcançado com o cumprimento das ações relativas à assistência farmacêutica no que concerne, por exemplo, na seleção, programação, aquisição, armazenamento, distribuição, dispensação de medicamentos e presença de serviços especializados (tais como, farmacovigilância, farmácia clínica e centro de informação de medicamentos). A avaliação do serviço de farmácia hospitalar tem sido objeto de estudos em diversos países, de forma a permitir a definição de estratégias que visem à melhor prestação de serviço 2,3 .

Desde a década de 1990 no Brasil, sociedades e profissionais da área de farmácia hospitalar têm apontado a necessidade de instrumentos que possibilitem avaliar esses serviços 4 . Devido à escassez de informação sobre os serviços de farmácia hospitalar, foi desenvolvido, na década passada, um projeto de abrangência nacional (Diagnóstico da Farmácia Hospitalar no Brasil), visando à avaliação de seus recursos materiais e humanos, serviços e atividades ${ }^{5}$. Objetivava-se deste modo, conhecer o cenário em que a farmácia hospitalar se encontrava, a fim de auxiliar a formulação de medidas para melhorias no setor 5 .

No entanto, a avaliação ampla de um serviço de farmácia hospitalar é uma tarefa complexa, devido às suas múltiplas atividades inter-relacionadas e à grande massa de dados usualmente requerida. Entre as técnicas estatísticas que podem ser utilizadas para tal encontra-se a análise de agrupamentos conhecida também como análise de cluster, que, baseada na similaridade entre objetos estudados, permite subdividi-los em um pequeno número de grupos mutuamente excludentes e não predefinidos ${ }^{6}$. Outra técnica de interesse é a análise de correspondência múltipla, que tem sido empregada na análise de grande volume de dados de variáveis categóricas, permitindo a redução dimensional dos atributos. Um dos resultados da análise de correspondência múltipla é a representação gráfica denominada mapa de correspondência, que possibilita visualizar possíveis relações entre categorias de variáveis e, portanto, a estrutura relacional entre as mesmas 6 . Assim, o presente estudo objetivou avaliar a existência de associações entre as va- riáveis relacionadas à assistência farmacêutica em serviço de farmácia hospitalar e caracterizar seus perfis no país, com base na utilização das técnicas estatísticas análise de correspondência múltipla e análise de agrupamentos. Desse modo, espera-se identificar um elenco conciso de variáveis para o processo de avaliação contínua dos serviços de farmácia hospitalar no país.

\section{Materiais e métodos}

\section{Análise de correspondência múltipla}

A análise de correspondência múltipla 6,7 é uma técnica multivariada capaz de analisar um conjunto grande de variáveis categóricas. Essa técnica é aplicada a uma matriz indicadora $\mathbf{Z}$, formada por códigos binários, em que nas linhas $(i)$ estão presentes os serviços de farmácia hospitalar e nas colunas $(j)$ as categorias das variáveis. Nessa matriz, cada categoria é codificada com " 1 " se estiver presente no serviço de farmácia hospitalar ou "0" caso contrário. A partir de Z, padronizações são realizadas por meio da frequência relativa marginal (massa) de linhas $\left(r_{i}\right)$ e colunas $\left(c_{j}\right)$, gerando a matriz de correspondência padronizada $\mathbf{G}$, conforme a equação 1 .

$g_{i j}=\frac{f_{i j}}{\sqrt{r_{i}} \sqrt{c_{j}}}$

Em que: $f_{i j}=\frac{z_{i j}}{N}, r_{i}=\sum_{j=1}^{p} f_{i j}, c_{j}=\sum_{i=1} f_{i j}$ para $1 \leq$ $i \leq n$ e $1 \leq j \leq p ; \mathrm{z}_{i j}=$ elementos de $\mathbf{Z} ; N=$ número total de elementos de $\mathbf{Z} ; r_{i}=$ massa de linhas; $c_{j}=$ massa de colunas.

Em seguida, utiliza-se um algoritmo de decomposição por valores singulares (DVS) para fatorar a matriz de correspondência padronizada $\mathbf{G}$ em três diferentes matrizes: duas de vetores singulares relativos aos serviços de farmácia hospitalar (linhas- $\mathbf{U}_{n x p}$ ) e as categorias (colunas- $\mathrm{V}_{p x p}$ ), e uma matriz quadrada $\left(\mathbf{D}_{p x p}\right)$, na qual a diagonal principal é composta por valores singulares associados a cada uma das linhas ou colunas, sendo esta matriz utilizada para o cálculo da inércia.

Um passo intermediário é calcular as coordenadas-padrão de linha e coluna por meio da divisão das matrizes $\mathbf{U}$ e $\mathbf{V}$ pela raiz quadrada de suas massas de linha $\left(r_{i}\right)$ e coluna $\left(c_{j}\right)$, respectivamente. Finalizando, as coordenadas principais são calculadas pelo produto das coordenadas-padrão de linha e coluna e seus respectivos valores singulares da matriz. As coordenadas principais contêm os valores das posições dos pontos-linha e pontos-coluna para cada uma das dimensões utilizadas para construir o mapa de correspondência. Desse modo, o mapa de correspondência é formado pela projeção das coordenadas prin- 
cipais de linha e/ou de coluna nas duas ou três dimensões (eixos) de maior inércia (maior contribuição para a variabilidade dos dados). A visualização do mapa permite verificar as associações existentes entre as categorias das variáveis com base na distância entre as mesmas: quanto mais próximas estiverem maior a probabilidade de associação. A interpretação do mapa é feita por meio de parâmetros como a inércia total, a contribuição relativa para a inércia e variáveis suplementares. A inércia total é a medida utilizada para indicar a variabilidade dos dados no espaço determinado pelas dimensões (eixos). A contribuição relativa para a inércia $\left(C t r_{j d}\right)$ é uma medida da variância do eixo dado por uma determinada linha (serviço de farmácia hospitalar) ou coluna (categoria) do mapa, indicando a importância da linha ou coluna para certa dimensão. Essa contribuição ${ }^{8}$ é calculada por meio de:

$C t r_{j d}=\frac{c_{j}\left(y_{j d}\right)^{2}}{\lambda d}$

Em que: $c_{j}=$ frequência relativa marginal ou massa da categoria $j ; y_{j d}=$ coordenada principal da categoria $j$ na dimensão $d ; \lambda_{d}=$ inércia na dimensão $d$. A contribuição relativa das variáveis é calculada como a soma das contribuições de suas categorias para a dimensão em análise, e são descartadas as variáveis cuja contribuição for inferior a $1 / Q$ (Q: número de variáveis) 8 .

As variáveis na análise de correspondência múltipla podem ser consideradas como ativas ou suplementares. As ativas são usadas para definir o espaço geométrico onde o conjunto de dados será avaliado, e fazem parte do cálculo da inércia e das coordenadas principais de linhas e colunas. Ao contrário, as suplementares não interferem nos cálculos da análise de correspondência múltipla, mas podem ser usadas para auxiliar na interpretação dos resultados do mapa de correspondência.

\section{Análise de agrupamentos}

A análise de agrupamentos ${ }^{6}$ (clusters) é um conjunto de técnicas multivariadas para construir grupos com base na similaridade que objetos (serviço de farmácia hospitalar) possuem, resultando em objetos os mais semelhantes possível dentro do grupo e distintos entre os grupos. Assim, os agrupamentos resultantes devem exibir elevada homogeneidade interna e heterogeneidade externa. Uma das finalidades da sua utilização é a redução de dados pela formação de subgrupos específicos e menores, por exemplo, quando o número de clusters não é conhecido.

O algoritmo de agrupamento hierárquico pode ser dividido entre dois métodos: aglomerati- vo e divisivo. No método aglomerativo ocorrem sucessivas junções dos objetos baseados na sua similaridade, e no divisivo ocorre a separação destes objetos a partir do conjunto inteiro de casos, com base em suas diferenças. O resultado é visualizado por um diagrama bidimensional, o dendrograma, em que a escala horizontal mostra os objetos de acordo com a sua similaridade, e a vertical indica o nível de similaridade 6 .

Algumas etapas são necessárias para a definição dos grupos, entre elas a escolha da medida de similaridade para indicar o quão próximos os objetos se encontram; do método de agrupamento hierárquico e do número de agrupamentos. Entre as medidas de similaridade está presente a distância Euclidiana comumente conhecida, que indica a maior similaridade entre dois objetos pela menor distância entre eles. Sobre o método de agrupamento hierárquico aglomerativo pode-se optar pelo tipo Ward, em que a seleção dos objetos em cada grupo é baseada em qual combinação irá minimizar a soma interna do quadrados das distâncias, promovendo assim uma menor variância entre os objetos agrupados 6,9.

Com relação à determinação do número de grupos, apesar de ser um procedimento subjetivo, pode-se optar pela análise do dendrograma observando quantos grupos parecem existir. Devido a essa subjetividade, alguns métodos foram desenvolvidos para auxiliar essa escolha, sendo a análise do comportamento no nível de fusão um deles. Nesse método, à medida em que se avança nos passos do algoritmo de agrupamento aglomerativo, o número de grupos vai diminuindo devido às fusões com semelhantes, e a distância entre eles vai aumentando, de modo que é possível observar pontos de "saltos" dos valores destas distâncias que indicam grupos distintos e, deste modo, o momento ideal de parada do algoritmo 9 .

\section{Dados}

O estudo utilizou variáveis do banco de dados do mencionado projeto Diagnóstico da Farmácia Hospitalar no Brasil, realizado entre 2002 e 2003. Os dados foram coletados em 250 serviços de farmácia hospitalar com mais de vinte leitos, por amostragem aleatória, em todas as regiões do país. As variáveis foram originalmente disponíveis em módulos, definidos como: caracterização geral do hospital; caracterização geral do serviço de farmácia hospitalar; logística de programação; logística de aquisição; logística de armazenamento; logística de distribuição; gerenciamento; seleção de medicamentos; farmacotécnica; informação; seguimento farmacoterapêutico; e ensino e pesquisa. Foram consideradas para a 
presente análise somente as variáveis que atendessem aos critérios: (i) número de respostas não preenchidas inferior a 5\%; (ii) número de respostas "não se aplica" inferior a 20\%; e (iii) para variáveis dicotômicas, pelo menos $5 \%$ de uma das respostas. Desse modo, das 224 variáveis do banco de dados original, somente 69 foram inicialmente selecionadas.

Em seguida, uma variável denominada "boas práticas de dispensação" foi construída baseando-se em oito variáveis dicotômicas originais do módulo logística de distribuição: responsável técnico durante a dispensação; instalações com boas condições higiênicas sanitárias; local limpo; equipamento de segurança para combater incêndio; identificação do setor de dispensação; produtos protegidos da ação da luz, umidade e temperatura; geladeira para a guarda de medicamentos; local para a guarda de medicamentos sujeitos a controle especial. Adotou-se uma categorização com base no número de respostas positivas, ou seja, 0-2 "sim" implicava boas práticas de dispensação "insatisfatória", 3-5 "sim" boas práticas de dispensação "regular" e 6-8 "sim" boas práticas de dispensação "satisfatória”.

Similarmente, outras 11 variáveis do módulo logística de armazenamento foram utilizadas na criação da variável adequabilidade de estocagem: área de estocagem em boa conservação, área de fácil limpeza, área está limpa, proteção contra roedores, ambiente com refrigeração, controle diário da temperatura ambiente, disposição do armazenamento correta e racional, manual para produtos com prazos de validade próximos ao vencimento, área para o armazenamento de produtos vencidos, medicamentos sujeitos a controle especial trancados, e produtos empilhados com segurança. Adotou-se também uma categorização com base no número de respostas positivas, ou seja, 0-4 "sim" implicava adequabilidade de estocagem "insatisfatória", 5-7 "sim" adequabilidade de estocagem "regular" e 8-11 "sim" adequabilidade de estocagem "satisfatória".

A descrição das 52 variáveis restantes pode ser consultada em Nascimento ${ }^{10}$, e a descrição pormenorizada das 224 variáveis originais pode ser consultada em Osório-de-Castro \& Castilho 5 .

As trinta variáveis selecionadas para a avaliação das atividades de assistência farmacêutica foram: tipo de hospital, número de leitos ativos, organograma no hospital, subordinação da farmácia, área da farmácia, período de funcionamento com farmacêutico, produtos com que trabalha, profissional responsável técnico, relação de produtos para orientar a compra, utilização da curva $A B C$, serviço de farmácia hospitalar fornece parecer técnico, sistema de registro de estoque, adequabilidade de estocagem, sistema de distribuição de medicamentos, conferência da prescrição, boas práticas de dispensação, manual de normas e procedimentos, planejamento de objetivos e metas, farmacêuticos com especialização, farmacêuticos com curso de atualização no último ano, farmacêuticos participantes de congressos no último ano, comissão de controle de infecção hospitalar, comissão de suporte nutricional, comissão de farmácia e terapêutica, relação de medicamentos padronizados, protocolo para uso de medicamentos específicos, atividades de farmacotécnica, fornece informação sobre medicamentos, literatura especializada, estágio em farmácia.

\section{Modelagem}

Inicialmente, foi realizada uma análise de correspondência múltipla com as 52 variáveis selecionadas, tomando-se por base uma matriz na qual as linhas correspondiam aos serviços de farmácia hospitalar e as colunas às categorias de variáveis. A seguir, as variáveis que apresentaram uma contribuição relativa para a inércia inferior ao limite usualmente recomendado ( $1 / Q$, em que $Q$ é o número de variáveis) 8 foram descartadas, e uma nova análise de correspondência múltipla foi então realizada com as variáveis remanescentes. As variáveis do módulo de "caracterização geral do hospital" e "caracterização geral do serviço de farmácia hospitalar” (Tabela 1) foram utilizadas como variáveis suplementares. Sendo identificada a relação entre as categorias por meio da análise do mapa de correspondência e da contribuição relativa das categorias para a inércia (Tabela 1). Adicionalmente, realizou-se uma análise de agrupamentos que teve como objetos os valores de coordenadas principais de linhas (serviço de farmácia hospitalar) das duas primeiras dimensões da análise de correspondência múltipla. Nessa análise de agrupamentos, optou-se pela distância Euclidiana como medida de similaridade e o número de agrupamentos foi definido com base na análise do dendrograma resultante e no comportamento de seu nível de fusão ${ }^{9}$. Todo o processamento foi realizado com os pacotes ca e stats do programa estatístico R versão 2.15.0 (The R Foundation for Statistical Computing, Viena, Áustria; http:// www.r-project.org).

\section{Resultados}

Do conjunto inicial de 250 serviços de farmácia hospitalar, 12 foram excluídos por terem mais de $30 \%$ de respostas ausentes. Assim, foram utilizados os dados de 238 serviços de farmácia hospi- 
Tabela 1

Trinta variáveis utilizadas na avaliação das atividades de assistência farmacêutica em 238 serviços de farmácia hospitalar. Brasil, $2002-2003$.

\begin{tabular}{|c|c|c|c|}
\hline \multirow[t]{2}{*}{ Variáveis } & \multirow[t]{2}{*}{ Categorias [\%] } & \multicolumn{2}{|c|}{ Contribuição relativa inércia } \\
\hline & & Dimensão 1 & Dimensão 2 \\
\hline \multicolumn{4}{|l|}{ Caracterização geral do hospital } \\
\hline Tipo de hospital & E1 [24,8]; E2 [6,8]; E3 [30,2]; E4 [8,8]; E5 [21,0]; E6 $[8,4]$ & - & - \\
\hline Número de leitos ativos & L? $[0,8] ;$ L1 [53,8]; L2 [38,7]; L3 [6,7] & - & - \\
\hline Organograma no hospital & O1 $[37,0] ;$ O2 $[63,0]$ & - & - \\
\hline Subordinação da farmácia & $\mathrm{S} ?[0,4] ; \mathrm{S} 1[8,0] ; \mathrm{S} 2[18,5] ; \mathrm{S} 3[5,5] ; \mathrm{S} 4[67,6]$ & - & - \\
\hline \multicolumn{4}{|l|}{ Caracterização geral do serviço de farmácia hospitalar } \\
\hline Área da farmácia & $\mathrm{A} 1[74,8] ; \mathrm{A} 2[18,9] ; \mathrm{A} 3[6,3]$ & - & - \\
\hline Período de funcionamento com farmacêutico & F1 $[37,4] ; F 2[50,4] ; F 3[12,2]$ & - & - \\
\hline Produtos com que trabalha & P1 $[7,1] ;$ P2 $[92,9]$ & - & - \\
\hline Profissional responsável técnico & RT? [0,4]; RT1 [51,7]; RT2 [47,9] & - & - \\
\hline \multicolumn{4}{|l|}{ Logística de programação } \\
\hline Relação de produtos para orientar a compra & RP1 [69,7]; RP2 [30,7] & 0,032 & 0,034 \\
\hline Utilização da curva ABC & $\mathrm{ABC}[11,8] ; \mathrm{ABC} 2[88,2]$ & 0,049 & 0,001 \\
\hline \multicolumn{4}{|l|}{ Logística de aquisição } \\
\hline Serviço de farmácia hospitalar fornece parecer técnico & PT? [0,8]; PT1 [16,8]; PT2 [82,4] & 0,033 & 0,016 \\
\hline \multicolumn{4}{|l|}{ Logística de armazenamento } \\
\hline Sistema de registro de estoque & RE? $[0,4] ;$ RE1 $[30,7] ; \operatorname{RE} 2[23,9] ;$ RE3 $[45,0]$ & 0,045 & 0,150 \\
\hline Adequabilidade de estocagem & AE? [0,4]; AE1 [17,2]; AE2 [38,7]; AE3 [32,4]; AE4 [11,3] & 0,037 & 0,210 \\
\hline \multicolumn{4}{|l|}{ Logística de distribuição } \\
\hline Sistema de distribuição de medicamentos & D1 $[35,3] ;$ D2 $[13,0] ;$ D3 $[51,7]$ & 0,051 & 0,014 \\
\hline Conferência da prescrição & $\mathrm{CP} ?[0,8] ; \mathrm{CP} 1[21,0] ; \mathrm{CP} 2[77,3] ; \mathrm{CP} 3[0,8]$ & 0,056 & 0,011 \\
\hline Boas práticas de dispensação & BPD1 $[26,9] ;$ BPD2 $[48,3] ;$ BPD3 $[24,8]$ & 0,064 & 0,056 \\
\hline \multicolumn{4}{|l|}{ Gerenciamento } \\
\hline Manual de normas e procedimentos & POP1 $[23,9] ;$ POP2 $[76,1]$ & 0,043 & 0,014 \\
\hline Planejamento de objetivos e metas & PL? [0,4]; PL1 [7,6]; PL2 [92,0] & 0,060 & 0,032 \\
\hline Farmacêuticos com especialização & FE? $[0,4] ;$ FE1 $[6,7] ;$ FE2 $[27,7] ;$ FE3 $[65,1]$ & 0,065 & 0,126 \\
\hline Farmacêuticos com curso de atualização no último ano & CA1 $[8,4] ;$ CA2 $[13,4] ;$ CA3 $[78,2]$ & 0,041 & 0,029 \\
\hline Farmacêuticos participantes de congressos no último ano & C1 $[8,0] ; C 2[18,5] ; C 3[73,5]$ & 0,054 & 0,122 \\
\hline Comissão de controle de infecção hospitalar & I? [0,4]; I1 [66,0]; I2 [33,6] & 0,033 & 0,026 \\
\hline Comissão de suporte nutricional & $\mathrm{N} ?[0,4] ; \mathrm{N} 1[10,5] ; \mathrm{N} 2[89,1]$ & 0,043 & 0,043 \\
\hline \multicolumn{4}{|l|}{ Seleção de medicamentos } \\
\hline Comissão de farmácia e terapêutica & CFT1 $[12,6] ;$ CFT2 $[87,4]$ & 0,065 & 0,026 \\
\hline Relação de medicamentos padronizados & PA1 $[53,8] ;$ PA2 $[46,2]$ & 0,045 & 0,023 \\
\hline Protocolo para uso de medicamentos específicos & PE1 $[9,7] ;$ PE2 $[90,3]$ & 0,045 & 0,009 \\
\hline \multicolumn{4}{|l|}{ Farmacotécnica } \\
\hline Atividades de farmacotécnica & Tec1 $[9,7] ;$ Tec2 $[90,3]$ & 0,025 & 0,034 \\
\hline \multicolumn{4}{|l|}{ Informação } \\
\hline Fornece informação sobre medicamentos & $\ln 1[44,5] ; \ln 2[55,5]$ & 0,033 & 0,016 \\
\hline Literatura especializada & LE1 $[13,0] ;$ LE2 $[87,0]$ & 0,038 & 0,002 \\
\hline \multicolumn{4}{|l|}{ Ensino e pesquisa } \\
\hline Estágio em farmácia & $\operatorname{EF} 1[19,3] ;$ EF2 $[80,7]$ & 0,044 & 0,002 \\
\hline
\end{tabular}

Nota: o ponto de interrogação (?) corresponde à categoria missing da variável. 
talar, sendo $24,8 \%$ hospitais públicos sem UTI $6,8 \%$ públicos com UTI, $30,2 \%$ privados sem UTI $8,8 \%$ privados com UTI, $21 \%$ filantrópicos sem UTI e $8,4 \%$ filantrópicos com UTI. A maior parte desses 238 hospitais é de pequeno porte $(53,8 \%)$ sendo $38,7 \%$ e $6,7 \%$ hospitais de médio e grande portes, respectivamente. Consequentemente, a maioria dos serviços de farmácia hospitalar possui a área da farmácia pequena $(74,8 \%)$, seguida de média $(18,9 \%)$ e grande $(6,3 \%)$.

A análise de correspondência múltipla foi inicialmente realizada com 52 variáveis, sendo 21 destas eliminadas após a análise de suas contribuições relativas para a inércia (Figura 1). Das 31 variáveis resultantes, como duas delas apresentavam a mesma distribuição (número de leitos e número de leitos ativos), optou-se pela retirada da variável número de leitos. Desse modo, a análise final foi realizada com trinta variáveis.

O fluxograma completo de seleção de variáveis pode ser visto na Figura 1, e a análise exploratória das trinta variáveis selecionadas e os seus respectivos valores de contribuição relativa para a inércia das dimensões 1 e 2 são apresentados na Tabela 1, a qual mostra que a dimensão 1 é formada principalmente pelas variáveis conferência da prescrição, boas práticas de dispensação, planejamento de objetivos e metas, farmacêuticos com especialização e comissão de farmácia e terapêutica (contribuições em torno de $6 \%$ para cada uma).

O mapa da análise de correspondência múltipla (Figura 2a) mostra que a inércia ajustada para as duas primeiras dimensões (eixos) é de 95\%, sendo $90,6 \%$ na primeira dimensão. Nesse mapa, estão presentes as categorias de variáveis ativas (em preto) e de variáveis suplementares (em negrito). Devido ao maior valor de inércia para a dimensão 1, observa-se um grande espalhamento das categorias sobre esta dimensão.

Na dimensão 1 à esquerda (Figura 2a) estão as categorias relacionadas à ausência de atividades e estruturas, enquanto que na região oposta (direita) há uma concentração de categorias mais desejáveis, como boas práticas de dispensação satisfatória (BPD1), conferência da prescrição pelo farmacêutico (CP1) e presença de planejamento de objetivos e metas (PL1). Próximo à origem das dimensões, ou seja, no centroide, situam-se as categorias intermediárias, de situação "regular", como por exemplo, sistema de registro de estoque por meio de ficha de prateleira (RE2) e adequabilidade de estocagem regular (AE2).

Para melhorar a visualização do mapa ampliaram-se as duas áreas marcadas na Figura 2a (Figuras 2b e 2c). A Figura 2b mostra as categorias relacionadas à ausência de atividades e estruturas dos serviços de farmácia hospitalar, sendo possí- vel relacioná-las com as categorias das variáveis suplementares: tipo de hospitais públicos, privados e filantrópicos sem CTI (E1, E3 e E5); número de leitos ativos até 50 leitos (L1); ausência de organograma no hospital (O2); subordinação da farmácia a outro setor não identificado (S4); área da farmácia até $50 \mathrm{~m}^{2}$ (A1); nenhum período de funcionamento com farmacêutico (F1); os produtos com que trabalha são medicamentos e outros materiais (P2); e profissional responsável técnico não ser o farmacêutico (RT2). Existe ainda nessa região uma associação entre quatro categorias de variáveis que ocupam praticamente o mesmo ponto no mapa: tipo de hospital público sem CTI (E1), área da farmácia até $50 \mathrm{~m}^{2}$ (A1), subordinação da farmácia a outro setor não identificado (S4) e ausência de organograma no hospital (O2). Outras duas categorias também possuem essa característica: número de leitos ativos até 50 leitos (L1) e tipo de hospital privado sem CTI (E3).

A Figura 2c mostra as categorias relacionadas à presença de atividades e estruturas dos serviços de farmácia hospitalar, podendo-se observar três grupos distintos: um próximo ao centroide, um intermediário e outro mais afastado. Próximo ao centroide (grupo 1), verifica-se uma associação entre as seguintes categorias suplementares tipo de hospital privado com CTI (E4) e período de funcionamento com farmacêutico de 1 a 9 horas (F2). Número de leitos ativos de 51 a 200 leitos (L2), com subordinação da farmácia à área administrativa (S2) e com farmacêutico como profissional responsável técnico (RT1). Presença de organograma no hospital (O1) com área da farmácia de 51 a $149 \mathrm{~m}^{2}$ (A2). No grupo 2, observase que a categoria suplementar subordinação da farmácia à área clínica (S1) não está associada a outra suplementar. Entretanto, essa região do mapa contém as categorias das variáveis ativas: conferência da prescrição pelo farmacêutico (CP1), presença de manual de normas e procedimentos (POP1), boas práticas de dispensação satisfatória (BPD1), adequabilidade de estocagem satisfatória (AE1), presença de literatura especializada (LE1) e oferece estágio em farmácia (EF1).

No grupo 3, existem associações entre as categorias suplementares tipo de hospital filantrópico com CTI (E6) e período de funcionamento com farmacêutico maior ou igual a 10 horas (F3); área da farmácia maior ou igual a $150 \mathrm{~m}^{2}$ (A3) e número de leitos ativos acima de 200 leitos (L3). Essa região contém as categorias de variáveis ativas relacionadas a atividades técnicas diferenciadas, como presença de comissão de farmácia e terapêutica (CFT1), e de comissão de suporte nutricional (N1), presença de protocolo para uso de medicamentos específicos (PE1), presença de 
Figura 1

Fluxograma da seleção de variáveis, dados coletados em 2002-2003 pelo projeto Diagnóstico da Farmácia Hospitalar no Brasil referentes a 238 serviços de farmácia hospitalar.

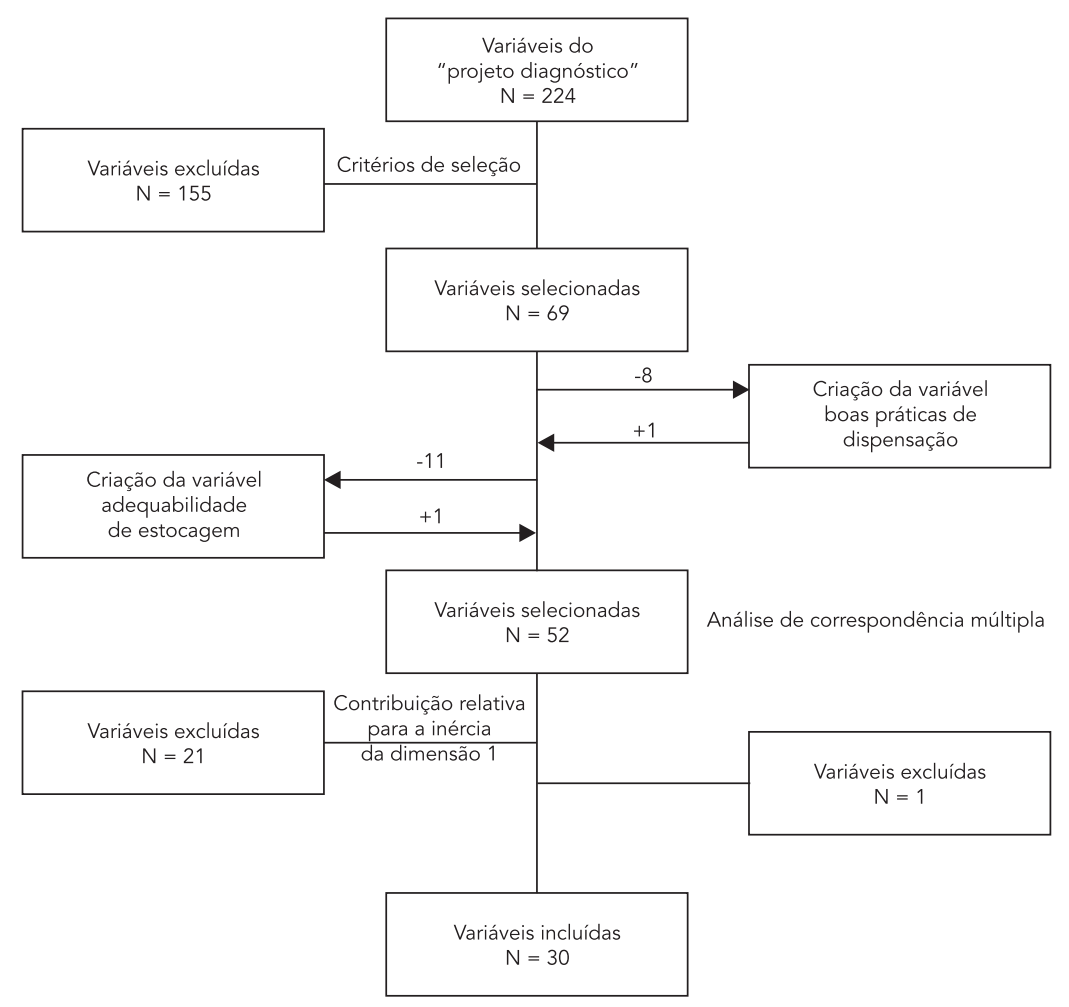

Nota: as duas variáveis criadas são boas práticas de dispensação e adequabilidade de estocagem. "Dimensão 1" se refere ao primeiro eixo do mapa de correspondência resultante da análise de correspondência múltipla. A contribuição relativa para a inércia é definida como a porção de inércia da dimensão dada pela categoria no mapa.

atividades de farmacotécnica (Tec1) e presença de planejamento de objetivos e metas (PL1). Possui também atividades relacionadas à educação do farmacêutico, como dois ou mais farmacêuticos com curso de atualização no último ano (CA1), dois ou mais farmacêuticos que participaram de congressos no último ano (C1) e dois ou mais farmacêuticos com especialização (FE1).

$\mathrm{Na}$ análise de agrupamento, o dendrograma da Figura 3 indica a existência de seis agrupamentos os quais dependeriam do porte dos hospitais no qual o serviço de farmácia hospitalar está inserido. $\mathrm{O}$ agrupamento referente ao serviço de farmácia hospitalar inserido em hospitais de grande porte com número de leitos ativos acima de 200 (grupo 6) é caracterizado por estar inserido em hospitais público com UTI (E2) e hospitais filantrópicos com UTI (E6). Esse agrupamento é destacado na segunda divisão na Figura 3, na qual estão presentes atividades e categorias mais desejáveis. As 11 variáveis determinantes na diferenciação dos demais grupos encontram-se listadas na Tabela 2.

\section{Discussão}

Existe uma preocupação crescente quanto à necessidade de avaliação dos serviços de farmácia hospitalar no país, e, consequentemente, quanto à necessidade de processos e instrumentos de avaliação consistentes e otimizados 3,11. No entanto, a maioria dos estudos de avaliação dos serviços de farmácia hospitalar baseia-se na análise do cumprimento de normas e atividades ou no desempenho de seus profissionais 12 , sem uma identificação da relação entre estas características. Para a presente análise, foram utilizadas du- 
Figura 2

Mapa de correspondência para uma análise de correspondência múltipla com trinta variáveis (oito suplementares - em negrito) e 238 serviços de farmácia hospitalar.

2a) Mapa completo

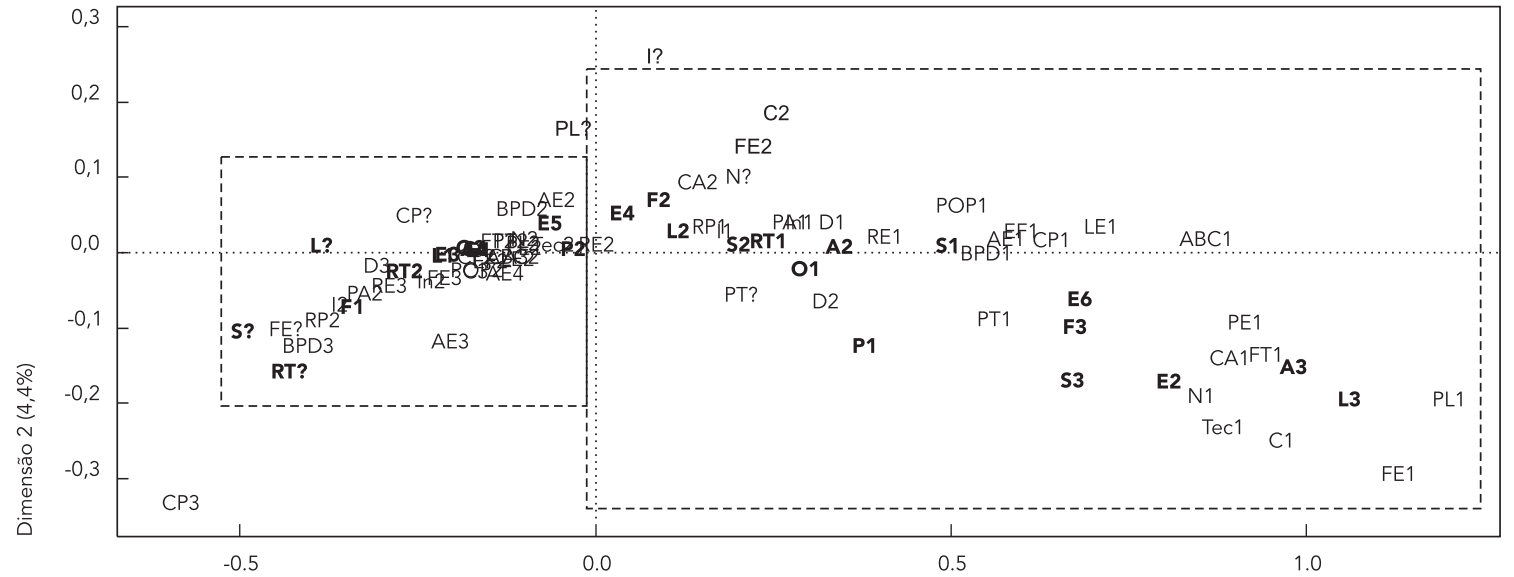

Dimensão $1(90,6 \%)$

2b) Região à esquerda da dimensão 1

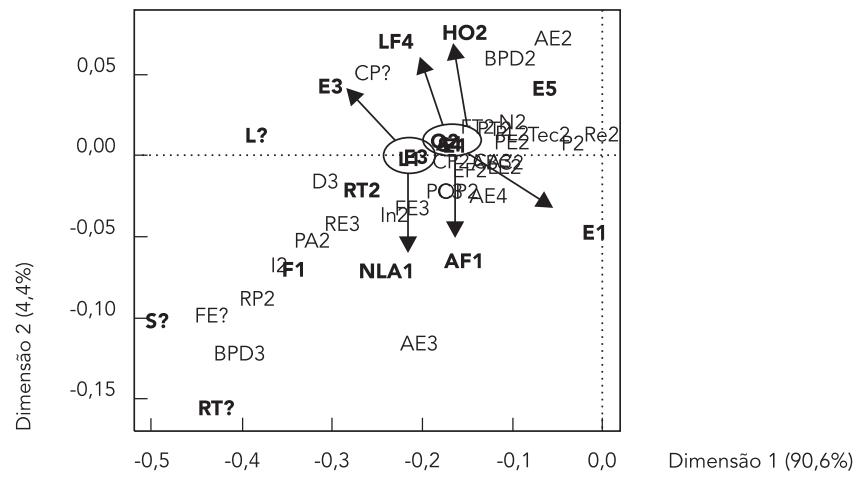

2c) Região à direita da dimensão 1

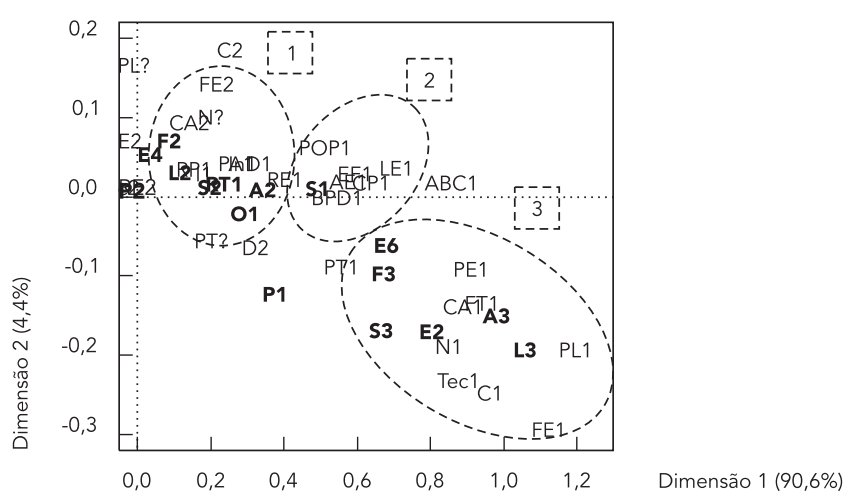

Nota 2a: a dimensão 1 explica 90,6\% da variabilidade dos dados.

Nota 2b: concentrando categorias referentes à ausência de atividades e estrutura como sistema de registro de estoque inexistente (RE3) e relação de produto para orientar a compra inexistente (RP2); e categorias menos desejáveis como boas práticas de dispensação insatisfatória (BPD3) e sistema de distribuição de medicamentos por dose coletiva (D3).

Nota 2c: concentrando categorias referentes à presença de atividades e estrutura como presença de manual de normas e procedimentos (POP1) e planejamento de objetivos e metas (PL1); e categorias mais desejáveis como boas práticas de dispensação satisfatória (BPD1) e sistema de registro de estoque informatizado (RE1).

as técnicas capazes de lidar com grandes bancos de dados compostos por variáveis categóricas: a análise de correspondência múltipla e a análise de agrupamentos. Essas técnicas têm sido, por exemplo, utilizadas para avaliar serviços de vacinação 13, para avaliar o perfil de mulheres na pós-menopausa e o uso da terapia de reposição hormonal 14, e para avaliar a acessibilidade a ser- viços de diagnóstico de tuberculose no país 15 , entre outros.

O banco de dados utilizado foi construído pelo projeto Diagnóstico da Farmácia Hospitalar no Brasil, financiado pela Secretaria de Assistência à Saúde do Ministério da Saúde, e que teve como motivação a carência de informações sobre a situação dos serviços de farmácia hos- 
Tabela 2

Variáveis mais importantes para a diferenciação entre os grupos de serviço de farmácia hospitalar na análise de agrupamentos (238 serviços e 30 variáveis). Brasil, 2002-2003.

\begin{tabular}{|c|c|c|}
\hline \multirow[t]{2}{*}{ Variável } & \multicolumn{2}{|c|}{ Grupos } \\
\hline & 1 & 2 \\
\hline Relação de produtos para orientar a compra & Presente & Ausente \\
\hline Adequabilidade de estocagem * & Regular * & Insatisfatória * \\
\hline \multirow[t]{2}{*}{ Boas práticas de dispensação * } & Regular * & Insatisfatória * \\
\hline & 3 & 5 \\
\hline Conferência da prescrição & Farmacêutico & Outro profissional \\
\hline Manual de normas e procedimentos & Presente & Ausente \\
\hline \multirow[t]{2}{*}{ Boas práticas de dispensação * } & Satisfatória * & Regular * \\
\hline & 4 & 6 \\
\hline Período de funcionamento com farmacêutico & 1 a 9 horas & Mais de 10 horas \\
\hline Conferência da prescrição & Outro profissional & Farmacêutico \\
\hline Manual de normas e procedimentos & Ausente & Presente \\
\hline Farmacêuticos com especialização & Nenhum & 2 ou mais \\
\hline Adequabilidade da estocagem * & Insatisfatória * & Satisfatória * \\
\hline
\end{tabular}

* Variáveis indicativas da qualidade da dispensação e do armazenamento.

Figura 3

Dendrograma dos serviços de farmácia hospitalar com a divisão de seis grupos.

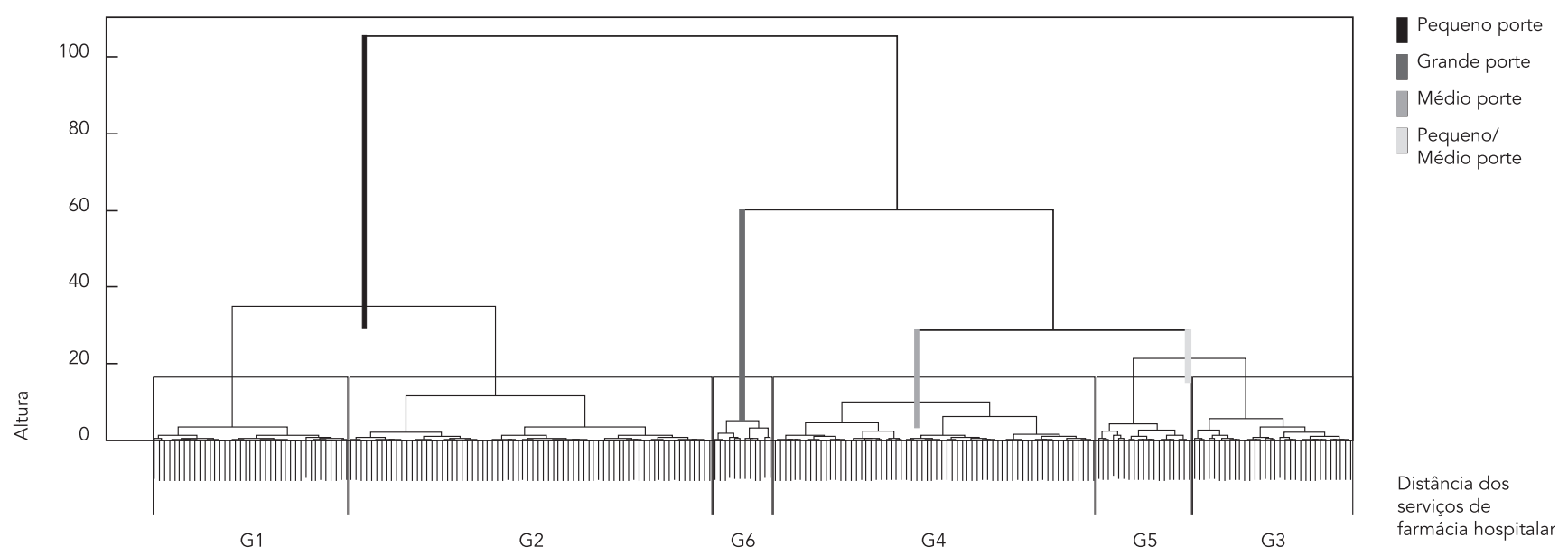

Nota: os grupos (G) 1 e 2 são compostos por serviços de farmácia hospitalar de pequeno porte (menor número de leitos). O grupo 6 é composto por serviços de farmácia hospitalar de grande porte (maior número de leitos). O grupo 4 é composto por serviços de farmácia hospitalar de médio porte (número de leitos médio) e os grupos 3 e 5 são compostos por serviços de farmácia hospitalar de médio e pequeno porte. 
pitalar no país. Esse projeto foi desenvolvido em âmbito nacional, incluiu unidades exclusivamente por seleção aleatória e foi baseado em entrevistas in loco com os responsáveis pelo serviço de farmácia hospitalar e do hospital onde este estava localizado. As entrevistas foram estruturadas com perguntas que resultaram, na sua maioria, em variáveis categóricas relativas às atividades desenvolvidas nas etapas da assistência farmacêutica 5 .

$\mathrm{Na}$ análise de correspondência múltipla, são observadas "nuvens" de pontos em um espaço multidimensional, que são projetadas em dimensões com melhor capacidade de caracterizar as distâncias entre os pontos. No presente estudo, essas distâncias foram basicamente representadas pela dimensão 1 , a qual concentrou praticamente toda a inércia (variabilidade) do mapa de correspondência. A análise dessa dimensão indica a clara separação dos serviços de farmácia hospitalar de acordo com as atividades de assistência farmacêutica. Assim, as categorias das variáveis analisadas apresentam um padrão ao longo da dimensão 1, indicando um "gradiente" entre os extremos, e esta dimensão caracteriza de maneira importante a estrutura dos serviços de farmácia hospitalar.

A região à direita nessa dimensão indica um melhor desempenho no cumprimento de atividades de assistência farmacêutica, e, consequentemente, sugere o fornecimento de um melhor serviço ao paciente. Essa região é caracterizada pela maior presença do farmacêutico (F3) e por um maior interesse e/ou incentivo na sua atualização profissional (FE1, CA1, C1), mostrando a importância destas características para o melhor desempenho das atividades do serviço de farmácia hospitalar. Sem dúvida, a relação entre a importância do conhecimento técnico do profissional responsável pela farmácia e uma resultante melhoria na utilização de medicamentos tem sido frequentemente enfatizada na literatura 16,17 .

Observou-se ainda que essa região (direita do mapa) estava relacionada ao aumento de tamanho (L3), do tipo (público - E1 e filantrópico - E6) e da complexidade de um hospital (presença de UTI), e à presença de um organograma do hospital (O1). Assim, na dimensão 1 é possíve observar que o serviço de farmácia hospitalar em hospitais de grande porte tem maior possibilidade de possuir atividades de assistência farmacêutica. Resultados similares são encontrados em estudos que indicam uma maior eficiência e um maior número de atividades da assistência farmacêutica desenvolvidas em serviço de farmácia hospitalar inserido em hospitais de maior porte 2,18 . Por exemplo, é sabido que farmacêuti- cos em hospitais maiores realizam atividades de monitoramento da terapia medicamentosa com mais frequência 2 .

As cinco variáveis que apresentaram maior contribuição relativa para a inércia da primeira dimensão (conferência da prescrição, planejamento de objetivos e metas, boas práticas de dispensação, comissão de farmácia terapêutica e farmácias com especialização) fazem parte dos módulos de gerenciamento e logística de distribuição, indicando serem estes componentes importantes para a formação da dimensão 1, e, consequentemente, para o cumprimento das atividades de assistência farmacêutica.

A análise de agrupamentos revelou seis grupos, diferenciados fundamentalmente pelos indicadores de desempenho introduzidos (adequabilidade de estocagem e boas práticas de dispensação) nos grupos 1 e 2 (ambos L1), a presença da relação de produtos para orientar a compra implica uma melhora nos níveis de adequabilidade e estocagem e boas práticas de dispensação de insatisfatória para regular. Com relação aos grupos 3 e 5 (L1 e L2, respectivamente), a presença de manual de normas e procedimentos e do farmacêutico na conferência da prescrição implica também uma mudança na boa prática de dispensação de regular para satisfatória. Os grupos 4 e 6 (L2 e L3, respectivamente) têm características de atividades similares, nas quais a presença de manual de normas e procedimentos, a presença do farmacêutico na conferência da prescrição, maior período de funcionamento com farmacêutico e a presença de farmacêuticos com especialização indicam uma mudança no nível de adequabilidade de estocagem de insatisfatória para satisfatória.

Por meio de um processo de consenso de especialistas (Delfos), Magarinos-Torres et al. ${ }^{4}$ utilizaram as opiniões de 22 profissionais de quatro regiões do país para construir indicadores de resultados para a farmácia hospitalar brasileira. Com base nessa consulta, foram criados vinte indicadores, que possuíam elevado índice de concordância. Entre as variáveis selecionadas com a análise de correspondência múltipla, 13 foram similares aos indicadores criados naquele estudo, a saber: relação de produtos para compra, serviço de farmácia hospitalar fornece parecer técnico, manual de normas e procedimentos, planejamento de objetivos e metas, farmacêuticos com especialização, farmacêuticos com curso de atualização no último ano, farmacêuticos participantes de congressos no último ano, comissão de controle de infecção hospitalar, comissão de suporte nutricional, comissão de farmácia e terapêutica, relação de medicamentos padronizados, fornece informação sobre 
medicamentos, e possui literatura especializa$d a$. Isso sugere que as informações das variáveis selecionadas no presente trabalho podem ser utilizadas para avaliar o serviço de farmácia hospitalar, sendo a análise de correspondência múltipla uma ferramenta útil na seleção de variáveis que expliquem o serviço de farmácia hospitalar em um contexto de otimização de tempo e recursos.

Cabe ressaltar que todas as variáveis utilizadas na construção da variável boas práticas de dispensação representam exigências da legislação vigente e, portanto, deveriam ser atendidas por um serviço de farmácia hospitalar que pretendesse assegurar a qualidade de suas atividades. Embora a técnica empregada não incorpore pesos às diferentes variáveis, adotou-se uma categorização com base no número de respostas positivas, ou seja, 0-2 "sim" implicava boas práticas de dispensação "insatisfatória", 3-5 "sim" boas práticas de dispensação "regular"; e 6-8 "sim" boas práticas de dispensação "satisfatória”. Além disso, o não cumprimento de itens como a presença do responsável no momento da dispensa- ção ou mesmo a indisponibilidade das condições ideais de armazenamento de medicamentos implica o risco potencial para a consecução do uso racional de medicamentos.

De modo geral, tanto a análise de correspondência múltipla quanto a análise de agrupamento mostraram que um maior cumprimento das atividades de assistência farmacêutica associase a variáveis relacionadas ao gerenciamento, principalmente à presença de planejamento; ao farmacêutico (maior tempo dedicado ao serviço de farmácia hospitalar e maior nível de treinamento), e ainda ao tamanho do hospital (maior número de leitos) e à sua complexidade (presença de CTI). Esses resultados foram obtidos por métodos quantitativos, extremamente úteis e necessários para analisar as variáveis que são relacionadas entre si, como é o caso das atividades de assistência farmacêutica, permitindo assim a construção de questionários mais concisos e informativos. Dessa forma, espera-se contribuir para a identificação de um elenco mais reduzido de variáveis para a avaliação contínua dos serviços de farmácia hospitalar no Brasil.

\section{Resumen}

El estudio tuvo como objetivo evaluar la existencia de asociaciones entre variables de servicios de farmacia hospitalaria. Se utilizaron 30 variables del proyecto Diagnóstico de Farmacia Hospitalaria en Brasil, relativas a la caracterización general del hospital, caracterización general del servicio de farmacia hospitalaria y etapas de la asistencia farmacéutica. Se utilizaron técnicas estadísticas como el análisis de correspondencia múltiple y el análisis de agrupamientos. La dimensión 1 del análisis de correspondencia múltiple explicó un 90,6\% de la variabilidad, diferenciando los servicios de farmacia hospitalaria conforme la presencia de actividades, sugiriendo así un eje de caracterización de la estructura de los servicios de farmacia hospitalaria. Los resultados indican la relación directa entre el cumplimiento de las actividades y el tipo de hospital y farmacéuticos con especialización. El análisis de agrupamientos identificó seis grupos relativos al porte del hospital, consiguiendo un mayor cumplimiento de actividades los servicios de farmacia hospitalaria en unidades de gran porte y con farmacéutico (mayor tiempo dedicado al servicio de farmacia hospitalaria y mayor nivel de experiencia). Se concluye que las técnicas fueron capaces de identificar las asociaciones y un elenco conciso de variables para una evaluación englobadora de los servicios de farmacia hospitalaria en el país.

Servicio de Farmacia en Hospital; Servicios

Farmacéuticos; Preparaciones Farmacéuticas 


\section{Colaboradores}

A. Nascimento, R. M. V. R. Almeida, S. R. Castilho e A. F. C. Infantosi participaram da pesquisa bibliográfica, análise e interpretação de dados, redação e revisão do texto.

\section{Agradecimentos}

Ao CNPq, pelo financiamento com bolsa de mestrado e, ao Núcleo de Assistência Farmacêutica (ENSP/Fiocruz) pela disponibilização do banco de dados utilizado neste trabalho.

\section{Referências}

1. Sociedade Brasileira de Farmácia Hospitalar. Padrões mínimos para farmácia hospitalar. Goiânia Sociedade Brasileira de Farmácia Hospitalar; 2007.

2. Pedersen CA, Schneider PJ, Scheckelhoff DJ. ASHP national survey of pharmacy practice in hospitals settings: Monitoring and patient education - 2009 . Am J Health Syst Pharm 2010; 67:542-58.

3. Messeder AM, Osorio-de-Castro CGS, Camacho LAB. Projeto Diagnóstico da Farmácia Hospitalar no Brasil: uma proposta de hierarquização dos serviços. Cad Saúde Pública 2007; 23:835-44.

4. Magarinos-Torres R, Osório-de-Castro CGS, Pepe VLE. Critérios e indicadores de resultados para a farmácia hospitalar brasileira utilizando o método Delfos. Cad Saúde Pública 2007; 23:1791-802.

5. Osório-de-Castro CGS, Castilho SR, organizadores. Diagnóstico da farmácia hospitalar no Brasil. Rio de Janeiro: Editora Fiocruz; 2004.

6. Hair JF, Tatham RL, Anderson RE, Black W. Análise multivariada de dados. 5a Ed. Porto Alegre: Book man; 2005

7. Greenacre M. Correspondence analysis in practice. 2nd Ed. Barcelona: Chapman and Hall/CRC; 2007.

8. Le Roux B, Rouanet H. Geometric data analysis from correspondence analysis to structured data analysis. Dordrecht: Kluwer Academic Publishers; 2004.

9. Mingoti SA. Análise de dados através de métodos de estatística multivariada: uma abordagem aplicada. Belo Horizonte: Editora da UFMG; 2005.

10. Nascimento A. Avaliação de farmácias hospitalares brasileiras utilizando análise de correspondência múltipla [Dissertação de Mestrado]. Rio de Janeiro: Universidade Federal do Rio de Janeiro; 2011.

11. Silva MJS. Avaliação da farmácia hospitalar em hospitais estaduais do Rio de Janeiro [Dissertação de Mestrado]. Rio de Janeiro: Escola Nacional de Saúde Pública Sergio Arouca, Fundação Oswaldo Cruz; 2010.
12. Penaforte TR, Foster AC, Silva MJS. Evaluation of the performance of pharmacists in terms of providing health assistance at a university hospital. Clinics 2007; 62:567-72.

13. Carvalho MS, Struchiner CJ. Análise de correspondência: uma aplicação do método à avaliação de serviços de vacinação. Cad Saúde Pública 1992; 8:287-301.

14. Aranha RN, Faerstein E, Azevedo GM, Werneck G, Lopes CS. Análise de correspondência para avaliação do perfil de mulheres na pós-menopausa e o uso da terapia de reposição hormonal. Cad Saúde Pública 2004; 20:100-8.

15. Scatena LM, Villa TCS, Ruffino Netto A, Kritski AL Figueiredo TMRM, Vendramini SHF, et al. Dificuldades de acesso a serviços de saúde para diagnóstico de tuberculose em municípios do Brasil. Rev Saúde Pública 2009; 43:389-97.

16. Kolawole OA, Pedersen CA, Schneider PJ, Smeenk DA. Perspectives on the attributes and characteristics of pharmacy executives. Am J Health Syst Pharm 2002; 59:278-81.

17. Zilz DA, Woodward BW, Thielke TS, Shane RR, Scott B. Leadership skills for a high-performance pharmacy practice. Am J Health Syst Pharm 2004; 61:2562-74.

18. Gupta SR, Wojtynek JE, Walton SM, Botticelli JT, Shields KL, Quad JE, et al. Association between hospital size and pharmacy department productivity. Am J Health Syst Pharm 2007; 64:937-44.

Recebido em 02/Ago/2012

Versão final reapresentada em 22/Out/2012

Aprovado em 28/Jan/2013 